\title{
Spontaneous chylothorax due to a left neck mass: Case report and literature review of a rare clinical entity
}

\section{Case Report}

A 36 year-old woman presented to A\&E with a two day history of painful left-sided neck swelling associated with odynophagia and left otalgia. There was no history of trauma, malignancy or contact with tuberculosis. She had experienced a previous similar episode 2 years previously for which her GP had referred her to ENT, but which had resolved by the time of her appointment.

Examination revealed diffuse swelling of the left neck extending to the chest wall with no discrete lymphadenopathy or collection. Blood tests showed mildly elevated inflammatory markers.

Ultrasound and CT imaging showed soft tissue inflammation with reactive lymph nodes and bilateral pleural effusions. Aspiration yielded milky fluid which was proved to be chyle (protein $35 \mathrm{~g} / \mathrm{L}$, cholesterol $2.62 \mathrm{mmol} / \mathrm{L}$, triglycerides $36.5 \mathrm{mmol} / \mathrm{L})$

Microscopy and culture of pleural fluid, sputum, and blood yielded no growth, no acid-fast bacilli, and no cytologically abnormal cells. The patient was investigated by the ENT and respiratory teams; no evidence of infection or malignancy was found.

The patient was treated with a low fat diet and her symptoms resolved within 3 days. At follow-up both the neck swelling and pleural effusions had completely resolved.

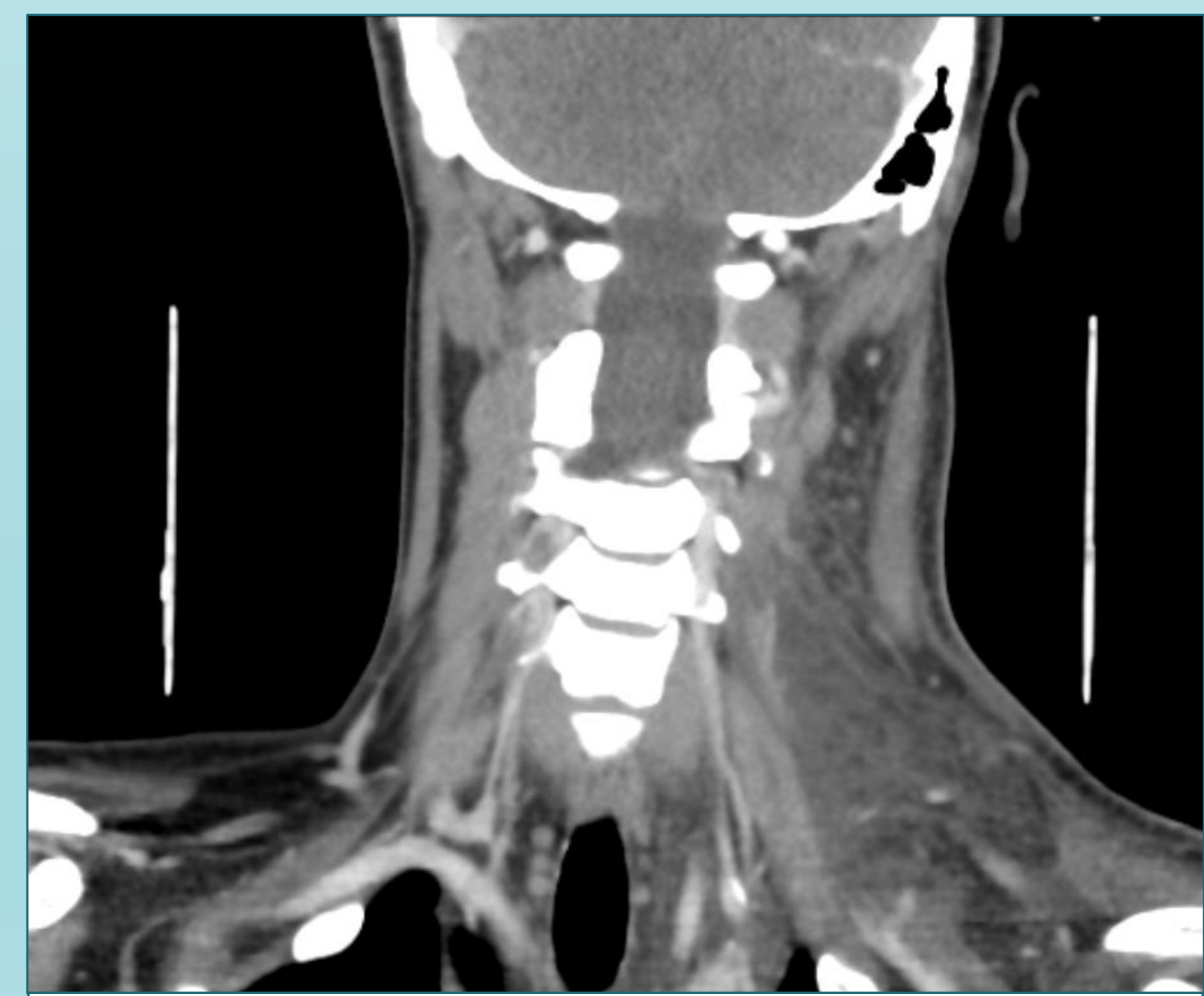

Figure 1: Coronal CT neck with contrast, showing diffuse inflammatory fat stranding in the left neck.

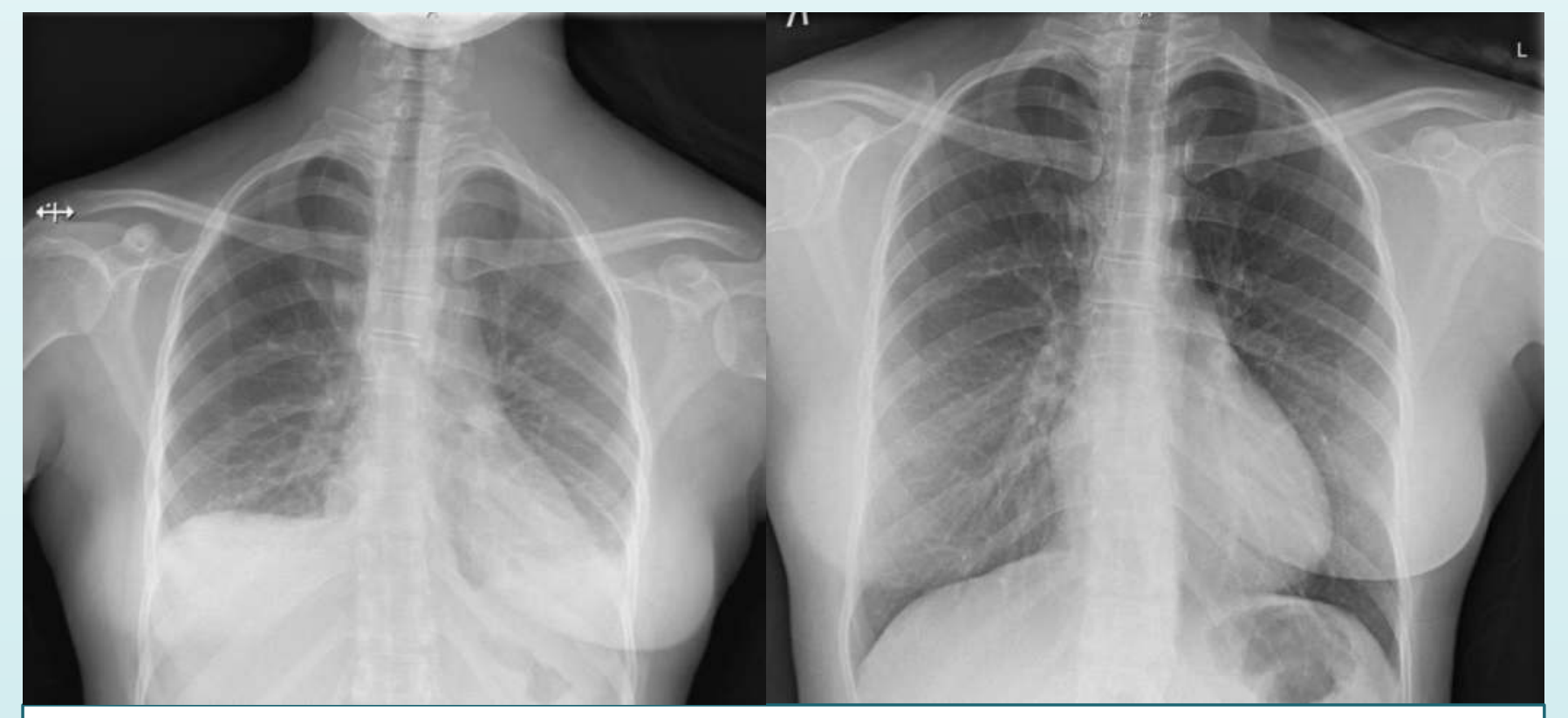

Figure 2: Left - admission chest X-ray shows small bilateral effusions. Right - repeat chest X-ray shows resolution.

\section{Discussion}

The most common causes of chyle leak are trauma, malignancy, and tuberculosis. All new pleural effusions should be aspirated, and a finding of chyle should prompt a search for a cause, particularly malignancy or atypical infection.

Spontaneous chylothorax and left or bilateral neck swelling in the absence of any definite cause has been described infrequently in the literature, always in women. In many cases the onset followed minor exertion - for instance after stretching.

In contrast to other causes of chylothorax which often require prolonged treatment with a low fat diet and/or surgical repair, the clinical course of described cases is of rapid resolution, though further episodes may occur.

\begin{tabular}{|l|l|l|l|l|l|l|}
\hline Year & Gender & Age & $\begin{array}{l}\text { Following } \\
\text { exertion }\end{array}$ & $\begin{array}{l}\text { Neck } \\
\text { swelling }\end{array}$ & Chylothorax & $\begin{array}{l}\text { Other } \\
\text { episodes }\end{array}$ \\
\hline $1960^{1}$ & Female & 35 & Yes & Left & Bilateral & No \\
\hline $1975^{2}$ & Female & 56 & Yes & Left & Bilateral & Yes \\
\hline $1984^{3}$ & Female & 45 & Yes & Left & Bilateral & No \\
\hline $1988^{4}$ & Female & 67 & No & Bilateral & Bilateral & No \\
\hline $2007^{5}$ & Female & 64 & Yes & Left & Bilateral & No \\
\hline $2012^{6}$ & Female & 40 & No & Left & Bilateral & No \\
\hline $2014^{7}$ & Female & 65 & Yes & Bilateral & Bilateral & No \\
\hline $2016^{8}$ & Female & 48 & No & Left & Left & Yes \\
\hline $2017^{9}$ & Female & 62 & Yes & Left & Bilateral & No \\
\hline 2018 & Female & 36 & No & Left & Bilateral & Yes \\
\hline
\end{tabular}

Table 1: Reported cases of spontaneous simultaneous neck swelling and chylothorax.

\section{Conclusions}

Although rare, the clinician should be alert to the possibility of spontaneous chyle leak in a patient presenting with a simultaneous neck swelling and pleural effusion. If other causes have been excluded conservative management is recommended. 